\title{
Essential fatty acid treatment prevents nerve ischaemia and associated conduction anomalies in rats with experimental diabetes mellitus
}

\author{
E.J.Stevens ${ }^{1}$, M.J.Lockett ${ }^{2}$, A.L. Carrington ${ }^{2}$ and D. R.Tomlinson ${ }^{1,2}$ \\ ${ }^{1}$ William Harvey Research Institute, Queen Mary and Westfield College, University of London, London, UK \\ ${ }^{2}$ Department of Pharmacology, Medical Sciences, Queen Mary and Westfield College, University of London, London, UK
}

\begin{abstract}
Summary. In rats with 6 weeks streptozotocin-diabetes there was a $53 \%$ reduction in sciatic nerve laser Doppler flux compared to controls $(p<0.01)$. Treatment of a parallel group of diabetic rats with evening primrose oil, by dietary admixture throughout the protocol, prevented this ischaemia (Doppler flux was $91 \%$ of evening primrose oil-treated controls and was not significantly different). There were no differences in systemic arterial pressure. In another experiment evening primrose oil markedly antagonised the development of exaggerated resistance to anoxic conduction failure in sciatic nerves from diabeticrats. The resistance to anoxia ofnervesfrom non-
\end{abstract}

diabetic rats was also reduced by evening primrose oil. These observations suggest that the sciatic nerves of diabetic rats with short-term streptozotocin-diabetes are markedly ischaemic and that thisischaemia isinvolvedin the development of increased resistance to anoxic/ischaemicconductionfailure in diabetic nerve. The findings also promote evening primrose oil as a potential treatment to prevent nerve ischaemia.

Key words: Diabetes mellitus, diabetic neuropathies, Doppler flux, essential fatty acids, gamma linolenic acid, anoxia, ischaemia, nerve conduction, rat, streptozotocin.
Hypotheses to explain acute or even chronic defects of nerve impulse conduction in diabetes mellitus fall into two categories: neurovascular insufficiency, leading to endoneurial ischaemia, or neurochemical abnormality - a direct registration of diabetes-induced biochemical disturbances to the axon or Schwann cell. The latter hypotheses are reviewed extensively elsewhere [1-3]. Support for the proposal of ischaemia comes from measurements of nerve blood flow and oxygen tension in both experimental and clinical diabetes. Thus, endoneurial nutritive flow is reduced by about $50 \%$ in experimental diabetes [4-6]. Endoneurial blood flow has not been measured in diabetic patients, but oxygen tension is low [7]. Not surprisingly reduced endoneurial oxygen tension has also been reported in the same diabetic animal model as that used for blood flow studies [4]. Further support comes from the demonstration that maintenance of persistent central hypoxaemia in non-diabetic rats causes a nerve conduction deficit of similar magnitude to that seen in diabetic rats breathing normal air $[8,9]$.

Pharmacological intervention aimed at improving nerve blood flow is therefore a rational prospect for prevention of early conduction abnormalities. Indeed, others have demonstrated prevention of slowed conduction by treatment of diabetic rats with a collection of drugs whose only likely unifying action is attenuation of vasoconstriction $[6,10,11]$. These are guanethidine (which re- duces noradrenaline release), lisinopril (an angiotensin converting enzyme inhibitor) and prazosin (an $\alpha_{1}$-selective adrenoceptor blocker).

There have also been several observations of attenuation of conduction deficits in diabetic rats by treatment with evening primrose oil [12-15]. Such treatment possibly also acts via production of vasodilators (see Discussion for proposed mechanisms). Accordingly, we designed the present study to measure a parameter of nerve blood flowlaser Doppler flux - and relate this to the development of exaggerated resistance to the conduction process observed in diabetic nerves exposed to abrupt ischaemia, hypoxia or anoxia. This particular conduction anomaly is associated with both clinical [16] and experimental [8] diabetes and may well develop as an adaptation to persistent incipient nerve ischaemia in diabetes. The study examined effects of essential fatty acidsupplementation-viaevening primrose oil-in control and streptozotocin-diabetic rats.

\section{Materials and methods}

\section{Experimental organization}

Two experiments were performed, each using male Wistar rats ( 300 350 g; 9-13 weeks of age; Charles River (UK) Ltd., Margate, Kent, UK). The aim of the first experiment was to measure any changes in 
sciatic nerve laser Doppler flux as an index of blood flow; the aim of the second was to study resistance to anoxic conduction failure. In each case the animals were assigned, at random, to four experimental groups, with diabetic and non-diabetic groups given diets enriched with either evening primrose oil or coconut oil. They were maintained under standard housing conditions with food (see below) and water available ad libitum.

Rats from both studies were fasted overnight prior to the induction of diabetes in two groups of each experiment by a single intraperitoneal injection of streptozotocin (Sigma, London, UK; $50 \mathrm{mg} / \mathrm{kg}$ (Study 1) or $65 \mathrm{mg} / \mathrm{kg}$ (Study 2), freshly dissolved in sterile $0.9 \%$ (weight/volume) NaCl). Two days later, blood samples were obtained by tail prick from the streptozotocin-treated rats and morning (nonfasted) blood glucose concentration measured by strip-operated reflectance photometry (Reflotest, Boehringer Corporation, London, UK). All animals had a morning blood glucose concentration of greater than $15 \mathrm{mmol} / \mathrm{l}$ and were included in the studies.

Immediately after confirmation of diabetes, one control group and one diabetic group were provided with a powdered diet (RM No. 1; Special Diet Services Ltd., Witham, Essex, UK) containing evening primrose oil (Efamol; Scotia Pharmaceuticals, Guildford, Surrey, UK) to comprise $5 \%$ of the dry weight $(w / w)$. The other groups were fed a diet containing $5 \%$ hydrogenated coconut oil to provide a control for the exaggerated fat intake associated with addition of evening primrose oil to the diet. These treatments were maintained for the full protocol, which comprised 6 weeks for the measurement of nerve Doppler flux (Study 1) and 5 weeks for the measurement of anoxic conduction block (Study 2). Body weight was recorded at least once a week. At the end of the protocol glucose in whole blood samples was measured by strip reflectance spectrometry (see above) and in Study 2 samples of whole blood were centrifuged $(9000 \mathrm{~g}$ for $1 \mathrm{~min}$ ) at room temperature to provide plasma for GOD-PERID glucose assay (test kit from Boehringer Mannheim, Mannheim, FRG) by conventional spectrophotometry.

\section{Sciatic nerve Doppler flux (Study 1)}

Rats were anaesthetised with chloral hydrate $(400-500 \mathrm{mg} / \mathrm{kg}$ i.p.). The left carotid artery was cannulated (PVC Intravenous Cannula outer diameter $1.34 \mathrm{~mm}$, inner diameter $1.00 \mathrm{~mm}$; Portex, Hythe, Kent, UK) and connected to a transducer (Type 4-327 L221; Bell \& Howell, Basingstoke, Hants., UK) for the measurement of systemic arterial pressure, which was displayed on a pen recorder (Multitrace 2; Electromed, St. Peter, Jersey). Via a small incision in the left flank the sciatic nerve was exposed and a thermocouple temperature probe inserted adjacent to, but not touching the nerve. The probe was connected to a fast response electronic thermometer (Comark Electronics, Rustington, Sussex, UK) and measurements of Doppler flux were made with the nerve at a temperature of $35.0 \pm 0.3^{\circ} \mathrm{C}$. This nerve temperature was maintained by placing the rat on a homeothermic blanket (Harvard Apparatus Ltd., Edenbridge, Kent, UK). Biofeedback from the rat was derived from a separate rectal temperature probe, and was set to maintain body temperature at $37.7 \pm 0.6^{\circ} \mathrm{C}$. A fibre optic flow probe (Type P4; tip diameter $0.85 \mathrm{~mm}$; Moor Instruments, Axminster, Devon, UK) was manoeuvred using an X-Y-Z micropositioner (Prior Scientific Instruments, Bishop's Stortford, Herts., UK) until it was just in contact with the nerve and a stable Doppler flux value was registered on the flow monitor (Type MBF3D; Moor Instruments). Measurements were made by withdrawal and repositioning of the probe on the same nerve. This was repeated until three measurements of similar proportions were obtained (in practice these were usually the first and only three) and the mean value over $2 \mathrm{~min}$ at one position was recorded. The whole process normally took less than $10 \mathrm{~min}$ from incision to completion. The rats were killed without recovery from anaesthesia.

Because the anaesthetic selected for this study gave relatively low arterial pressures in all groups (see Results), we obtained further data from a parallel study of control and diabetic rats (given no oil supplementation) using a different anaesthetic procedure. These animals were anaesthetised initially with halothane $\left(4 \%\right.$ in $\mathrm{O}_{2}$ for induction, $2-2.5 \%$ for maintenance) and a left jugular venous catheter inserted. This was used for infusion of a mixture (in $0.9 \% \mathrm{NaCl}$ ) of $9 \mathrm{mg} / \mathrm{ml}$ alphaxalone and $3 \mathrm{mg} / \mathrm{ml}$ alphadolone acetate (Saffan; Pitman-Moore Ltd, Uxbridge, Middx., UK). The manufacturer's solution was diluted 1 in 3 with $0.9 \% \mathrm{NaCl}$ and an initial dose of $4 \mathrm{mg} / \mathrm{kg}$ (body weight) of this dilution given on removal of the rats from the halothane source; the solution was infused slowly as the halothane was breathed off. The anaesthesia was maintained by continuous infusion of the same solution of alphaxalone/alphadolone at a rate of $12 \mathrm{mg} / \mathrm{h}$. All other aspects of the measurement procedure were identical to that described above.

\section{Resistance to anoxic conduction failure in vitro (Study 2)}

At the end of the protocol rats were killed by a blow to the head, bled from the throat and the left sciatic nerve was removed rapidly and mounted between two suction electrodes in Krebs-Henseleit bicarbonate buffered saline $(118 \mathrm{mmol} / 1 \mathrm{NaCl} ; 4.8 \mathrm{mmol} / 1 \mathrm{KCl} ; 25 \mathrm{mmol} / \mathrm{l}$ $\mathrm{NaHCO}_{3} ; 1.2 \mathrm{mmol} / 1 \quad \mathrm{KH}_{2} \mathrm{PO}_{4} ; 1.2 \mathrm{mmol} / 1 \quad \mathrm{MgSO}_{4} ; 2.5 \mathrm{mmol} / \mathrm{l}$ $\mathrm{CaCl}_{2}$ ), containing $0.5 \mathrm{mmol} / \mathrm{l}$ myo-inositol and $5 \mathrm{mmol} / \mathrm{l}$ glucose at $37^{\circ} \mathrm{C}$ and continuously gassed with $95 \% \mathrm{O}_{2}: 5 \% \mathrm{CO}_{2}$. This was done in a thermoregulated purpose-built tissue bath, which was continuously perfused with bathing fluid at a rate of $0.5 \mathrm{ml} / \mathrm{min}$. Electrical stimulation was applied to the proximal end of the preparation and the resulting compound action potentials (CAPs) recorded on a digital storage oscilloscope (Type 1421; Gould Instruments, Ilford, Essex, UK) via a differential pre-amplifier (DAM-5 A; W-P Instruments Inc., New Haven, Conn., USA). Supramaximal stimulation $(10 \mathrm{~V})$ was applied for a 30 -min stabilization period $(0.5 \mathrm{~Hz}, 0.1 \mathrm{~ms}$ duration) with hard copy recordings plotted on an $\mathrm{X}-\mathrm{Y}$ pen recorder every $10 \mathrm{~min}$. This was done to ensure that the preparation was capable of propagating identical CAPs in the absence of anoxia. After stabilization, stimulation was stopped and the bathing medium removed to be replaced with medium of the same composition but which had been previously gassed with a mixture of $5 \% \mathrm{CO}_{2}$ and $95 \% \mathrm{~N}_{2}$. This gas mixture was also supplied to the organ bath, and stimulation recommenced for a further $40 \mathrm{~min}$, with hard-copy recording at 10 -min intervals.

\section{Statistical analysis}

All data are presented as arithmetic mean \pm 1 SD. Statistical analysis of diabetes and treatment effects was carried out by one way analysis of variance (ANOVA). Where the F ratio gave $p<0.05$, and where there was homogeneity of variance (Cochrans \& Bartlett Box [17], $p>0.05$ ), comparisons between individual group means were made using Duncan's Multiple Range Test at significance levels of $p=0.05$ and $p=0.01$.

\section{Results}

\section{Animals}

Throughout the course of both studies, the body weight of control rats increased by about $3-3.5 \mathrm{~g} \cdot \mathrm{rat}^{-1} \cdot \mathrm{day}^{-1}$ whereas, at the end of the protocol, the body weight of the diabetic rats was slightly lower than the initial value (Table 1). There were no differences between either dietary oil in effects on body weight. Blood glucose data were similar in the two studies and are given in Table 1. Treatment with evening primrose oil had no effect on the general severity of diabetes since animal body weight and final plasma glucose concentration were not significantly altered in either study. 
Table 1. Body weight and blood glucose data for rats of both studies

\begin{tabular}{ll} 
Body weight $(\mathrm{g})$ & $\begin{array}{l}\text { Glucose concentra- } \\
\text { tion (mmol/l) }\end{array}$ \\
\hline At start At death & $\begin{array}{l}\text { In whole In plasma } \\
\text { blood }\end{array}$
\end{tabular}

\begin{tabular}{lllll}
\hline Study 1 & & & & \\
Control CO & $325 \pm 9$ & $501 \pm 36$ & $5.2 \pm 0.5$ & $\mathrm{NE}$ \\
Control EPO & $320 \pm 10$ & $495 \pm 51$ & $5.4 \pm 0.8$ & $\mathrm{NE}$ \\
Diabetic CO & $318 \pm 9$ & $338 \pm 32$ & $>25$ & $\mathrm{NE}$ \\
Diabetic EPO & $323 \pm 11$ & $370 \pm 32$ & $>25$ & $\mathrm{NE}$ \\
Study2 & & & & \\
Control CO & $309 \pm 15$ & $463 \pm 44$ & $6.5 \pm 1.6$ & $7.2 \pm 1.2$ \\
Control EPO & $307 \pm 11$ & $484 \pm 28$ & $6.1 \pm 1.5$ & $6.3 \pm 1.0$ \\
Diabetic CO & $318 \pm 6$ & $310 \pm 42$ & $>25$ & $40.1 \pm 6.4$ \\
Diabetic EPO & $309 \pm 16$ & $282 \pm 52$ & $>25$ & $39.2 \pm 8.9$ \\
\hline
\end{tabular}

Data are mean \pm 1 standard deviation. In Study 1 each group contained 8-9 rats and in Study 2 there were 10 rats per group. CO, Coconut oil-treated rats; EPO, evening primrose oil-treated rats; NE, not estimated

\section{Sciatic nerve Doppler flux}

Figure 1 shows individual animal data points together with group means and standard deviations. Comparison of the two coconut oil-treated groups showed a massive reduction in Doppler flux in the sciatic nerves of the diabetic rats. Treatment of non-diabetic rats with evening primrose oil did not significantly increase their sciatic nerve Doppler flux. Fluxes in the nerves of evening primrose oil-treated diabetic rats were only marginally lower than those of either non-diabetic group, indicating substantial prevention of the diabetes-associated ischaemia. Values for mean systemic arterial pressure are also given in Figure 1. There were no differences between the groups, indicating that increased neurovascular resistance was the main determinant of the reduced Doppler flux in the coconut oil-fed diabetic rats.

In the separate study with rats anaesthetised with alphaxalone/alphadolone the mean arterial pressures ( $\mathrm{mm}$ $\mathrm{Hg} \pm 1 \mathrm{SD})$ were $107.0 \pm 10.6(n=9)$ for controls and $108.0 \pm 7.5(n=5)$ for diabetics. These were associated with sciatic nerve Doppler fluxes (arbitrary units \pm 1 SD) of $376 \pm 40$ for controls and $157 \pm 23$ for diabetics. Thus, although chloral hydrate anaesthesia caused hypotension, it did not reduce sciatic nerve Doppler fluxes in either control or diabetic rats, compared to rats anaesthetised with alphaxalone/alphadolone. Furthermore, there were substantially reduced sciatic nerve fluxes in the diabetic rats irrespective of the anaesthetic used.

\section{Resistance to anoxic conduction failure}

These data are presented in Figure 2. The preparations from diabetic rats treated with coconut oil showed the established pattern of increased resistance to anoxic conduction failure, when compared with the controls treated with coconut oil. Comparison of the effects of the two oils indicates a marked effect of the evening primrose oil on both groups. The rate of decline of the CAP amplitude during anoxia was increased in the evening primrose oil-treated

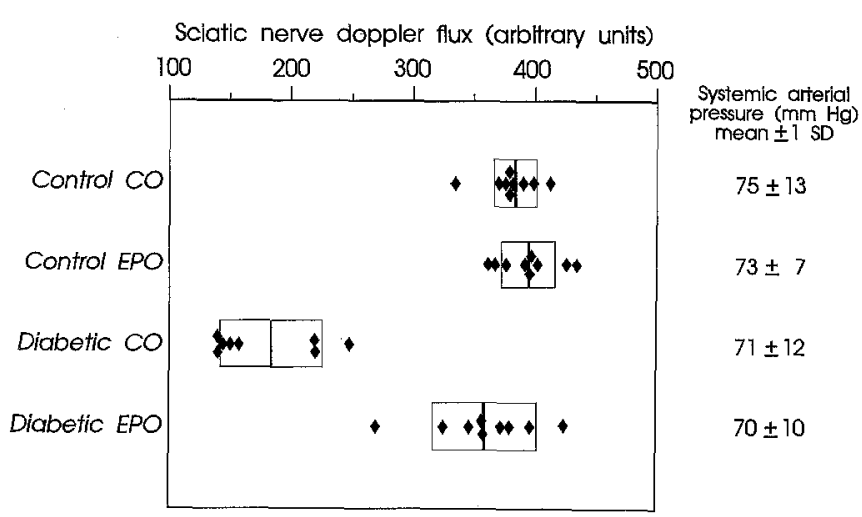

Fig.1. Effects of diabetes and of treatment with either coconut oil (CO) or evening primrose oil (EPO) on sciatic nerve laser Doppler flux and systemic arterial pressure. Individual points show nerve Doppler flux for each rat, bars indicate group means and the boxes show \pm 1 SD

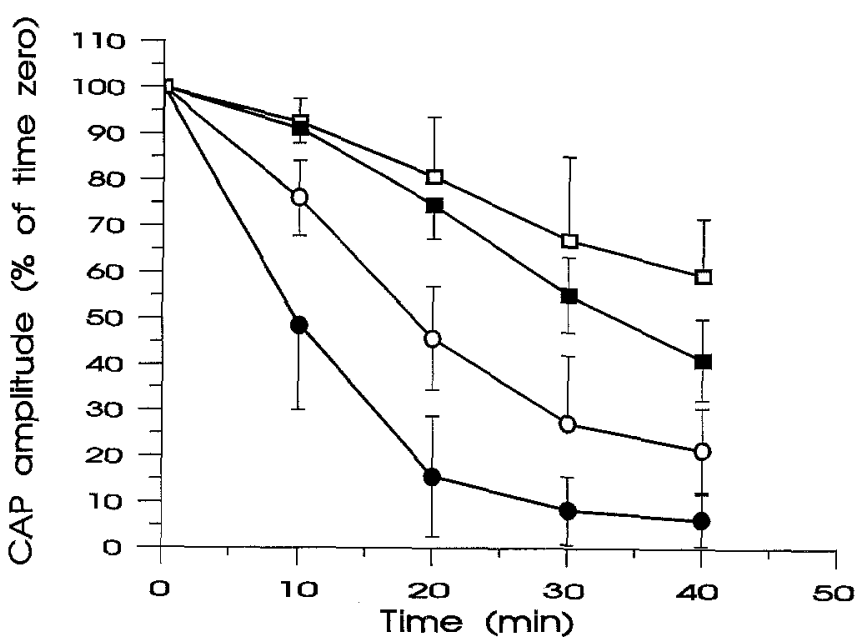

Fig. 2. Resistance to anoxic conduction failure in nerves from the four groups comprising the second study. Square symbols represent nerves from diabetic rats and circles those from non-diabetic rats; filled symbols indicate evening primrose oil-treated groups and open symbols those given coconut oil. Limits are $\pm 1 \mathrm{SD}$. At $\mathrm{t}_{40} \mathrm{~min}$ all group mean amplitudes differed at $p<0.05$. CAP, Compound action potential

diabetic group, bringing their performance closer to that of the coconut oil-treated controls. At $t_{40}$ the amplitude of the action potentials from evening primrose oil-treated diabetic nerves was mid-way between that of the coconut oil-treated control and diabeticgroups and was statistically significantly different from both $(p<0.05)$. The effect of evening primrose oil treatment on the nerves of control rats was even more startling, with a marked increase in decay rate relative to that seen in the nerves of the coconut oiltreated controls. At $t_{40}$ the action potential amplitude of nerves from the evening primrose oil-treated control rats was significantly different from that of all other groups.

\section{Discussion}

The treatment of rats with streptozotocin caused weight loss and hyperglycaemia, features characteristic of unrestrained Type 1 (insulin-dependent) diabetes mellitus. 
There was, however, no attenuation of glycaemia associated with evening primrose oil treatment. The most dramatic finding of this study is the prevention of a massive fall in sciatic nerve Doppler flux in diabetic rats by treatment with evening primrose oil. However, due consideration must be given to the limitations imposed on interpretation by the technique used. Laser Doppler flux is not a measure of blood flow; it is a parameter of the average velocity of moving particles passing the probe at a point in time. As such, its major determinants are erythrocyte velocity and number. It is, therefore, proportional to blood flow, but a linear relationship between the two cannot be guaranteed. However, another study applied Doppler flux monitoring and whole nerve blood flow measurements from $\left[{ }^{14} \mathrm{C}\right]$ iodoantipyrine in the same animals and reported a good agreement between the two over a flow range of 0.1 to $0.45 \mathrm{ml} \cdot \mathrm{min}^{-1} \cdot \mathrm{g}^{-1}$ [18]. These authors present a scatterplot of data from the two methods and, although some points deviate from the regression line by as much as $50 \%$, the deviations are random in the mid to low range of the plot. Given this validation of the Doppler flux technique, we suggest that the large difference in flux seen here in nerves of control and diabetic rats (on coconut oil) reflects a substantial difference in whole nerve blood flow. Doppler flux makes no discrimination between cells passing through epineurial and endoneurial vessels and it cannot differentiate nutritive (capillary) flow from nonnutritive flow. Thus, neither this method nor the use of labelled iodoantipyrine would allow altered values to be registered to either epineurial or endoneurial flow. Approximately $50 \%$ of whole nerve flow passes through the endoneurium [18]. In our study nerve Doppler flux was reduced in coconut oil-treated diabetic rats to $47 \%$ of the value recorded in controls. For this to occur without any reduction in endoneurial flow would require cessation of flow to the epineurium and perineurium. This is unlikely and the reduction of flux seen in the nerves of the diabetic coconut oil-fed rats is of a similar proportion - with reference to controls - to that seen in the same model by direct techniques, such as $\mathrm{H}_{2}$ clearance $[4,6]$ which is specific for nutritive flow. We suggest, therefore, that the data indicate a reduction in sciatic nerve blood flow in diabetic rats and that some of the reduction may have been in nutritive flow. Treatment with evening primrose oil prevented the low Doppler flux in the nerves of diabetic rats; this may have been achieved by shunting through non-nutritive vessels, but increased nutritive flow may have contributed.

The rats were hypotensive under chloral hydrate anaesthesia, but there was no bias between controls or diabetics. Use of an anaesthetic (alphaxalone/alphadolone) giving normal systemic arterial pressure gave a similar difference in nerve Doppler flux between control and diabetic rats, indeed the values were similar to those from the coconut oil-fed rats under chloral hydrate. This is surprising since peripheral nerve is not thought to autoregulate its blood flow $[19,20]$ and the higher systemic arterial pressure might have been expected to give a higher flux measurement. However, it is possible that the hypotension under chloral hydrate anaesthesia caused selective loss of vasomotor tone, decreasing vascular resistance dis- proportionately in peripheral nerve and maintaining flow despite decreased systemic arterial pressure.

Treatment with evening primrose oil also attenuated the exaggerated resistance to anoxic conduction failure in diabetic nerves in vitro, but there was an even more marked effect in the nerves from non-diabetic rats given evening primrose oil. This was not matched by an effect on nerve Doppler flux in non-diabetic rats. It is possible that exaggerated resistance to abruptly enforced anoxia, hypoxia or ischaemia develops in diabetes as an axonal adaptation to the incipient endoneurial hypoxia in the nerve trunks of the extremities. There may, however, be another mechanism which is manifest in both control and diabetic rats and which is not related to nerve blood flow.

Evening primrose oil is rich in oleic, linoleic and gamma-linolenic acid whilst coconut oil contains little linoleic and no gamma-linolenic acid [21]. The biological activity of this treatment is probably not due solely to the presence of a single active constituent, but may depend on several components [22]. Treatment with evening primrose oil probably enhances the synthesis of arachidonic acid by the conversion of linoleic acid through gamma-linolenic and dihomo-gamma-linolenic acids. Increased availability of arachidonate, derived from evening primrose oil may be directly responsible for prevention of nerve ischaemia by stimulating the production of vasodilator prostanoids. We have found deficient basal prostacyclin release in sciatic nerves of diabetic rats to be prevented by evening primrose oil treatment [23]. Increased prostaglandin $E_{1}$ production from gamma-linolenic acid [24] has been established in mesenteric vascular tissue [25] although it remains to be demonstrated in peripheral nerve. Prostaglandin $E_{1}$ seems to have an important role in the maintenance of normal neuronal function. Blockade of prostaglandin $E_{1}$ synthesis is associated with a reduction in nerve action potential amplitude in the rat, and this abnormality can be corrected by prostaglandin $E_{1}$ administration [26]. Recent studies have shown that a structural analogue of prostaglandin $E_{1}$ can prevent the acute deficit in motor nerve conduction velocity (MNCV) when administered to streptozotocin-diabetic rats $[27,28]$. Prostaglandin $\mathrm{E}_{1}$ might modulate biochemical events increasing nerve conduction or might improve the endoneurial microcirculation by dilating arterioles and inhibiting platelet aggregation, hence preventing the development of any MNCV deficit related to ischaemic endoneurial hypoxia $[4,29]$. Co-administration, to diabetic rats, of the cyclo-oxygenase inhibitor, flurbiprofen prevents the attenuation of exaggerated resistance to acute anoxic conduction failure by evening primrose oil [30]. This supports strongly an involvement of cyclooxygenase-derived products in this effect of evening primrose oil.

In conclusion, we suggest that reduced sciatic nerve Doppler flux in diabetic rats indicates possible ischaemia, which may be prevented by treatment with evening primrose oil. This reduced Doppler flux and its prevention are associated with clear changes in nerve conduction. In this study we report attenuation of resistance to acute anoxia and other studies have highlighted prevention of slowed conduction velocity. These observations strengthen the 
possibility of an involvement of ischaemia in the development of acute nerve conduction disturbances in diabetes.

Acknowledgements. Financial support for this reseatch was provided by a grant from Ono Pharmaceuticals to The William Harvey Research Institute (EJS), a project grant from the British Heart Foundation (ALC), an undergraduate bursary from the Medical Research Council (MJL) and a vacation grant from Scotia Pharmaceuticals for further support of MJL. We wish to thank Scotia Pharmaceuticals for the donation of the Efamol and coconut oil preparations.

\section{References}

1. Greene DA, Lattimer SA, Sima AAF (1988) Are disturbances of sorbitol, phosphoinositide, and $\mathrm{Na}^{+}-\mathrm{K}^{+}$-ATPase regulation involved in pathogenesis of diabetic neuropathy? Diabetes 37: 688-693

2. Greene DA, Lattimer SA, Sima AAF (1988) Pathogenesis and prevention of diabetic neuropathy. Diabetes Metab Rev 4: 201-221

3. Greene DA, Sima AAF, Pfeifer MA, Albers JW (1990) Diabetic neuropathy. Annu Rev Med 41:303-317

4. Tuck RR, Schmelzer JD, Low PA (1984) Endoneurial blood flow and oxygen tension in the sciatic nerves of rats with experimental diabetic neuropathy. Brain 107:935-950

5. Monafo WW, Eliasson SG, Shimazaki S, Sugimoto H (1988) Regional blood flow in resting and stimulated sciatic nerve of diabetic rats. Exp Neurol 99: 607-614

6. Cameron NE, Cotter MA, Low PA (1991) Nerve blood flow in early experimental diabetes in rats: relation to conduction deficits. Am J Physiol 261: E1-E8

7. Newrick PG, Wilson AJ, Jakubowski JA, Boulton AJM, Ward JD (1986) Sural nerve oxygen tension in diabetes. Br Med J 293: $1053-1054$

8. Low PA, Schmelzer JD, Ward KK, Yao JK (1986) Experimental chronic hypoxic neuropathy: relevance to diabetic neuropathy. Am J Physiol 250: E94-E99

9. Smith WJ, Diemel LT, Leach RM, Tomlinson DR (1991) Central hypoxaemia in rats provokes neurological defects similar to those seen in experimental diabetes mellitus: evidence for a partial role of endoneurial hypoxia in diabetic neuropathy. Neuroscience $45: 255-259$

10. Cameron NE, Cotter MA, Robertson S (1992) Angiotensin converting enzyme inhibition prevents development of muscle and nerve dysfunction and stimulates angiogenesis in streptozotocindiabetic rats. Diabetologia 35: 12-18

11. Cameron NE, Cotter MA, Ferguson K, Robertson S, Radcliffe MA (1991) Effects of chronic $\alpha$-adrenergic receptor blockade on peripheral nerve conduction, hypoxic resistance, polyols, $\mathrm{Na}^{+}$$\mathrm{K}^{+}$-ATPase activity, and vascular supply in STZ-D rats. Diabetes 40: $1652-1658$

12. Julu POO (1988) Essential fatty acids prevent slowed nerve conduction in streptozotocin diabetic rats. Diab Comp 2: 185-188

13. Tomlinson DR, Robinson JP, Compton AM, Keen P (1989) Essential fatty acid treatment-effects on nerve conduction, polyol pathway and axonal transport in streptozotocin diabetic rats. Diabetologia 32: 655-659

14. Cameron NE, Cotter MA, Robertson S (1991) Essential fatty acid diet supplementation: effects on peripheral nerve and skeletal muscle function and capillarization in streptozocin-induced diabetic rats. Diabetes 40: 532-539
15. Lockett MJ, Tomilnson DR (1992) The effects of dietary treatment with essential fatty acids on sciatic nerve conduction and activity of the $\mathrm{Na}^{+} / \mathrm{K}^{+}$pump in streptozotocin-diabetic rats. Br J Pharmacol 105: 355-360

16. Daube JR, Dyck PJ (1984) Neuropathy due to peripheral vascular diseases. In: Dyck PJ, Thomas RK, Lambert EH, Bunge R (eds) Peripheral neuropathy. $1^{\text {st }}$ Edn. pp 1458-1478

17. Sokai RR, Rohif FJ (1981) Biometry. 2nd edn. W.H. Freeman and Company, New York

18. Rundquist I, Smith QR, Michel ME, Ask P, Oberg PA, Rapoport SI (1985) Sciatic nerve blood flow measured by laser Doppler flowmetry and [14 C]iodoantipyrine. Am J Physiol 248: 311-317

19. Low PA, Tuck RR (1984) Effects of changes of blood pressure, respiratory acidosis and hypoxia on blood flow in the sciatic nerve of the rat. I Physiol 347:513-524

20. Sundqvist T, Oberg PA, Rapoport SI (1985) Blood flow in rat sciatic nerve duxing hypotension. Exp Neurol 90: 139-148

21. Manku MS (1983) A comparison of gas liquid chromatography and high performance liquid chromatography methods for determining fatty acid composition of evening primrose (Oenothere biennis) and soyabean oil. J Chromatog Sci 21: 367-369

22. Myher JJ, Marai A, Kuksis A (1977) Acylglycerol structure of peanut oils of different atherogenic potential. Lipids 12:775-785

23. Stevens EJ, Carrington AL, Tomlimson DR (1992) Prostacyclin release in experimental diabetes: effects of evening primrose oil. Br J Pharmac 107:276P

24. Horrobin DF, Manku MS, Huang YS (1984) Effects of essential fatty acids on prostaglandin biosynthesis. Biomed Biochim Acta 43: S114-S120

25. Watanabe Y, Manku MS, Jenkins DK, Mitchell JA, Horrobin DF (1987) The effects of short term dietary supplementation with evening primrose oil or safflower oil on fatty acid and prostaglandin release from perfused mesenteric vascular beds in spontaneously hypertensive rats. J Am Coll Nutr 6: 447-453

26. Horrobin DF, Durand LG, Manku MS (1977) Prostaglandin E1 modifies nerve conduction and interferes with local anaesthetic action. Prostaglandins 14: 103-108

27. Yasuda H, Sonobe M, Hatanaka I et al. (1988) A new prostaglandin E1 analogue (TFC-612) prevents a decrease in motor nerve conduction velocity in streptozocin-diabetic rats. Biochem Biophys Res Comm 150: 225-230

28. Yasuda H, Sonobe M, Yamashita M et al. (1989) Effect of prostaglandin $E_{1}$ analogue TFC 612 on diabetic neuropathy in streptozocin-induced diabetic rats: comparison with aldose reductase inhibitor ONO 2235. Diabetes 38: 832-838

29. Dyck PJ (1989) Hypoxic neuropathy: does hypoxia play a role in diabetic neuropathy ? New Engl J Med 320: 57-60

30. Cotter MA, Cameron NE, Dines KC, Maxfield E, Robertson S, Cox D (1992) The effect of evening primrose oil on nerve conduction in diabetic rats depends on product(s) of cyclooxygenase-mediated gamma-linolenic acid metabolism. Diabet Med 9 [Suppl 1] A12 (Abstract)

Received: 5 October 1992 and in revised form:13 January 1993

Professor D.R. Tomlinson

Department of Pharmacology

Medical Sciences

Queen Mary and Westfield College

Mile End Road

London E1 4NS

UK 